\title{
Inferring the goal of an approaching agent: a human-robot study
}

\author{
Patrizia Basili ${ }^{1}$ \\ Stefan Glasauer ${ }^{1}$ \\ Markus Huber ${ }^{1}$ Omiros Kourakos ${ }^{2}$ \\ Tamara Lorenz 3 \\ Thomas Brandt ${ }^{4}$ \\ Sandra Hirche ${ }^{2}$
}

\begin{abstract}
The ability to infer intentions and predict actions enables coordinating of one's own actions with those of another human and allows smooth and intuitive interaction. The aim to achieve equally effective human-robot interactions is a crucial aspect of current robotic studies. Thus, we assume that studying human-human interaction provides valuable insights allowing to implement mutual intention recognition and action prediction in robotic systems. A common scenario of interaction, be it in everyday life or in an industrial setting, is that two or more agents share the same workspace and perform tasks without interference. If humans are involved, the robots should act sufficiently predictable to enable the human to attribute goals and predict motion trajectories. In the present work, we first analyzed how well a human recognizes the goal of another person entering the room, and whether this ability is deteriorated by concealing gaze direction of the other person. In a second setup, the same experiment was repeated by replacing the approaching person with a wheeled robot. On average, the distance at which subjects predicted the goal of the approaching agent was approx. $4 \mathrm{~m}$ and depended on subject and goal position, but not on the type of agent. However, goal attribution showed a considerable proportion of errors for the robot (19\%), much less for a human with hidden gaze direction $(6 \%)$, and almost none for a human with visible gaze $(1 \%)$. Thus, our subjects apparently decided on the goal of the approaching agent without taking into account the reliability of directional cues, thus resulting in more errors. In a human-robot setting, such wrong predictions about robotic behavior may easily lead to dangerous situations. For smooth and safe interaction, it is therefore important to ameliorate the predictability of robotic actions.
\end{abstract}

\section{INTRODUCTION}

When sharing the same working or living area, humans are intuitively attentive to the presence and the actions of other persons. To prevent interfering with each other, they implicitly negotiate and coordinate their actions. Underlying this behavior is the ability to infer and understand the intentions of other people and thus predict their future behavior. The neural basis for this ability has been linked to the observation that patterns of brain activation while

\footnotetext{
*This work is supported by the DFG cluster of excellence CoTeSys

${ }^{1}$ Patrizia Basili, Markus Huber, Stefan Glasauer are with the Center for Sensorimotor Research Ludwig-MaximiliansUniversität München Marchioninistr 2381377 München, Germany \{p.basili,markus.huber,s.glasauer dlrz.uni-muenchen.de

${ }^{2}$ Omiros Kourakos, Tamara Lorenz, Sandra Hirche are with the Institute of Automatic Control Engineering, Technische Universität München (CCRL-519) CCRL - CoTeSys Central Robotics Laboratory Barer Str. 21 80333 Mnchen, Germany

${ }^{3}$ Tamara Lorenz is with the Institute of Experimental Psychology, Ludwig-Maximilians-Universität München

${ }^{4}$ Thomas Brandt is with the Chair of Clinical Neurosciences, LudwigMaximilians-Universität München Marchioninistr. 15, 81377 München, Germany
}

observing another agent's action reveal a replay of own motor programs and intention networks [1]-[3]. It is assumed that the other agent's behavior is simulated internally to infer the underlying intention and thus appropriate reactions for successful interaction [4]. One of the predominant theories explaining such behavior is the so called simulation theory [5], which explains how we can build a theory of mind, a theory about what other people think, feel, or intend to do [6]. According to the simulation theory, the mental state of other individuals is represented based on one's own mindset and perspective and it can be inferred even without verbal communication. An everyday situation of this predictive capability is to infer the immediate goal of another person sharing our workspace. To investigate this behavior, we performed human-human experiments, in which the test subject had to indicate whether another person, who entered the room, was heading towards himself or towards another target. Previous experiments on human locomotion (e.g., [7]) have shown that changes in gaze and head direction anticipate changes in movement trajectory. We therefore assumed that observation of head and gaze may improve goal recognition. Consequently, we also tested the effect of hiding gaze and head direction on goal recognition. In humanrobot scenarios, research on intention recognition focuses on the robots' capability to interpret human intentions [8], [9], but there are only few studies investigating how well humans attribute and recognize intentions of robotic agents [10]. Since mutual action understanding is key to seamless interaction, we investigated whether or not we can equally well recognize the goal of a mobile robot using the same experiment. Due to the predictability of motion trajectories highly depends on their kinematics, we used the previously measured human trajectories for the robot, so that both experiments were comparable. Thus, we exploit the fact that human locomotion can be approximated by the motion of a nonholomic system (a wheeled robot) [11]. The methods and results of the present study can be used to test and improve human aware motion planners [12]-[14] towards intuitive mutual intention recognition, which in turn helps to increase the safety of human-robot interaction and workspace sharing.

\section{EXPERIMENTAL SETUP}

Experiments were performed in the $17 \mathrm{~m}^{2}$ kitchen of CoTeSys Central Robotics Laboratory with a total of 14 subjects. They reported normal or corrected-to-normal vision, and were unaware of the purpose of the experiment. A marker-based infrared motion tracking system (Visualeyez II, PhoeniX Technologies Incorporated) was used to record 

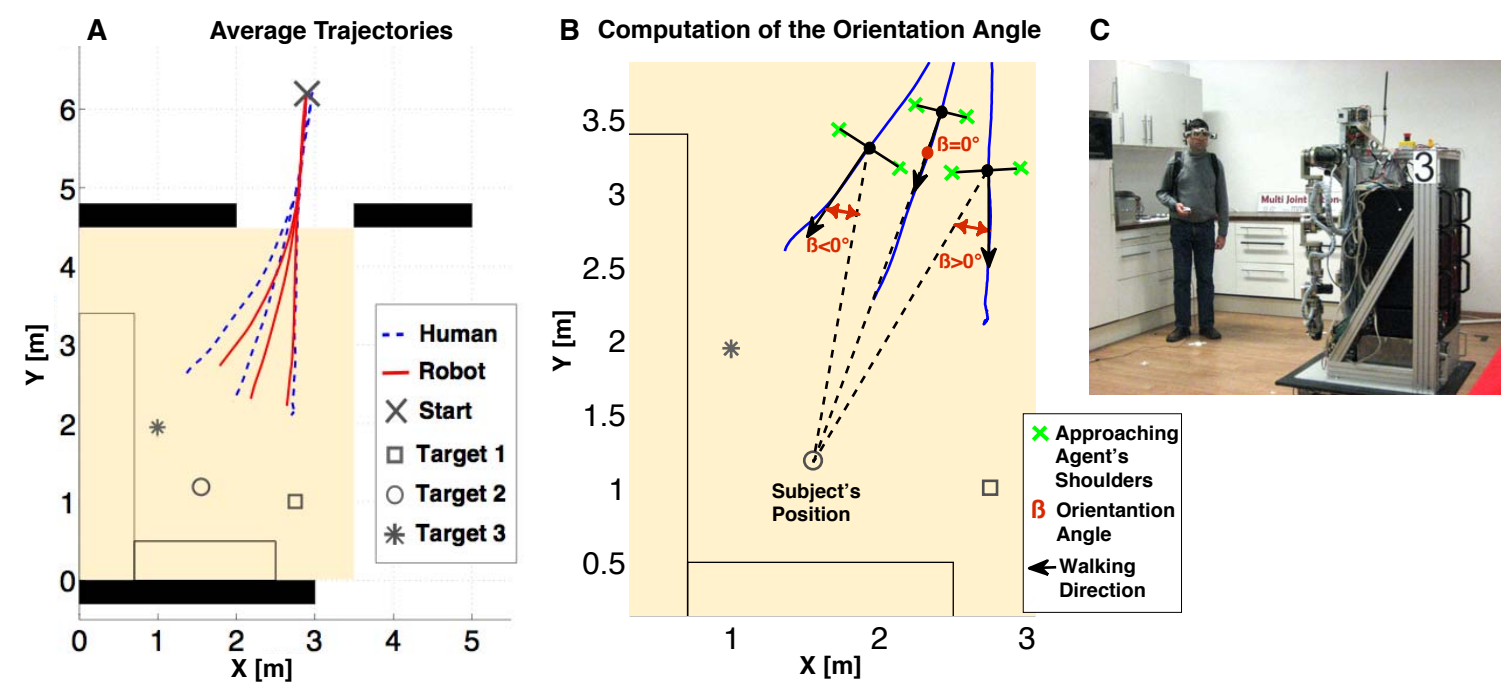

Fig. 1. A: Average human and robot trajectories measured and performed in the experiments. B: Definition of the orientation angle at the moment of goal attribution. C: Example of a human-robot experimental session. Here, the robot is not heading towards the subject.

TABLE I

DISTANCES BETWEEN STARTING POSITION AND TARGETS AND Between the 3 Targets. SeE Also Fig.1-A.

\begin{tabular}{ll}
\hline Target Positions & Distance $[\mathrm{m}]$ \\
\hline Start - Target 1 & 5.17 \\
Start - Target 2 & 5.16 \\
Start - Target 3 & 4.63 \\
Target 1 - Target 2 & 1.21 \\
Target 2 - Target 3 & 0.94 \\
\hline
\end{tabular}

the trajectories of the approaching person (first scenario, see sections II-A and II-B) or approaching robot (second scenario, see section II-C). The approaching agent, who always started from the same position, passed through a door to enter the kitchen (see Fig.1-A) in order to reach one of three predefined target positions (defined as T1, T2 and T3, see Fig.1-A). Two LED markers were placed on the shoulders of the approaching person or robot, in order to track their position and orientation. A subject had to indicate as soon as possible whether or not the agent's goal was to approach the subject by pressing the corresponding button on a standard two-button mouse (see Fig.1-C), which was connected to the tracking system. Thus, chance level was at 50\%. Subjects' positions varied between the 3 targets. The approaching person or robot was visible from each of the 3 target positions. The subjects were allowed to press the button multiple times if they changed their goal attribution during the approach of the agent. The position of the approaching person or robot was recorded together with the button press at the moment of goal attribution. In all the experiments the approaching agent entered the kitchen and moved five times towards each of the three target positions (15 trajectories in total). The order of targets to approach was randomized but the same for all the subjects. Distances between starting position and targets are listed in table I.

\section{A. Human-Human Scenario without Gaze}

Six male subjects (23-35 years old) attended one experimental session of approximately $7 \mathrm{~min}$ duration. The first two subjects were standing at target 1 (see Fig.1-A), subject 3 and 4 at target 2 while the remaining two were placed at target 3 . The same approaching person (male, 37 years old) performed in all the experiments. He wore dark sunglasses to cover his eyes and a scarf covering his neck in order to conceal gaze and head direction. To be comparable with the low robot speed, the approaching person walked slowly and kept the orientation of trunk and head aligned with his walking direction.

\section{B. Human-Human Scenario with Gaze}

The human-human experiments were repeated with the same subjects for an approaching person without sunglasses or scarf and with natural trunk and neck movements.

\section{Human-Robot Scenario}

Eight subjects (3 female, 23-36 years old) without prior knowledge of the robotic system participated in the experiment, which lasted approximately 15 min per subject. Subjects 1-3 were standing on target position 1 , subjects $4-6$ on target 2 and the remaining two on target 3 . The mobile robot [15] was built within the CoTeSys cluster at the Technische Universität München, Germany. The trajectories performed by the robot were based on previously measured trajectories of the human-human experiments but adapted to the larger dimension of the robot. Each trajectory was approximated by fitting it with a polynomial of degree $n$, where $n$ was typically chosen to be 12 . It was then transferred to the robot by sending the degree of the polynomial and the coefficients. In addition, the wheels of the robot were covered to prevent the subject from determining the motion direction of the robot from their orientation. The average human and robot trajectories are depicted in Fig.1-A. 


\section{DATA ANALYSIS}

The data was analyzed using the Matlab software (The Mathworks, Natick, MA). From the raw data, we extracted the position and velocity of the agent $300 \mathrm{~ms}$ before the subject pressed a button to indicate the inferred goal of the agent. The delay of $300 \mathrm{~ms}$ was introduced to account for reaction times [16]. For the further analysis, we only used correct responses. We considered several parameters: (1) the distance between the approaching agent and the subject; (2) the duration between the subject's decision and the agent reaching the goal; (3) the orientation of the approaching agent at the moment of decision. To define the start of the movement, we extracted the velocity profile of the approaching person or robot and determined the instance when the velocity exceeded $0.25 \mathrm{~m} / \mathrm{s}$ in human-human experiments (corresponding to a movement duration of $0.4 \mathrm{~s}$ ) or 0.2 $\mathrm{m} / \mathrm{s}$ in human-robot experiments (movement duration 1.3s). The orientation angle of the approaching agent (person or robot) at the moment of goal recognition was computed with respect to the subject, such that an orientation angle of zero degrees means that the approaching agent was heading straight toward the subject (see Fig.1-B). In the humanhuman experiments, the orientation angle was defined as average of the current angle and the previous 9 angles to compensate for the shoulders' movements while walking. For statistical analysis, we used repeated measures ANOVA (analysis of variance) and paired t-tests with a significance level of $p<0.05$. For the two human-human experiments, the ANOVA had two within-subject factors (target with 3 levels, gaze with 2 levels) and one between-subject factor (subject position, 3 levels). To compare human and robot approach, we chose to use the no-gaze data. Thus, the resulting ANOVA consisted of one within-subject factor (target, 3 levels) and two between-subject factors (subject position with 3 levels, agent with two levels, i.e., human or robot).

\section{RESULTS}

The percentage of correct goal prediction for all three scenarios is given in table II (chance level 50\%). A significant difference between the two human-human scenarios (one sided t-test, $p=0.035$ ) and between the human-human without visible gaze and the human-robot scenario (one sided t-test, $p=0.0034$ ) was found. In the following, a detailed comparison between the two human-human scenarios with and without gaze visibility is presented in section IV-A. The human-human and the human-robot scenario are compared in section IV-B.

\section{A. Human-Human Experiments with Visible and without Visible Gaze}

A representative example of one of the human-human experimental session with covered gaze and neck movements of the approaching person is depicted in Fig.2-A. The subject was standing at target 1 and thus the trajectories of the approaching person are shorter for $\mathrm{T} 1$. The sinusoidal deviations in the trajectories are a typical pattern in human
TABLE II

CORRECT AND ERRONEOUS GOAL ATTRIBUTION (CHANCE LEVEL 50\%). ALL DECISIONS (ALL BUTTON PRESSES) WERE CONSIDERED.

\begin{tabular}{llll}
\hline \multirow{2}{*}{ Prediction } & \multicolumn{3}{c}{ Scenario } \\
\cline { 2 - 4 } & Gaze & No Gaze & Robot \\
N Trials & 90 & 90 & 120 \\
Correct [\%] & 98.9 & 93.55 & 81.25 \\
Error [\%] & 1.1 & 6.45 & 18.75 \\
\hline
\end{tabular}

locomotion with oscillations corresponding to steps. An example of human-human goal recognition in relation to the velocity profiles two human-human experiments can be seen in Fig.3 for the same subject. Each session consisted of 15 trajectories, one session for visible gaze (Fig.3-A) and one for the no-gaze (Fig.3-B) scenario. For this subject, the moment of goal recognition was more variable and often occurred earlier when the gaze direction of the approaching person was visible. We therefore investigated whether the distance between starting position and position of goal attribution was affected by gaze visibility. While there was no effect of gaze on distance over the group of subjects, a repeated measures ANOVA with the standard deviation of distance as dependent variable revealed a significant main effect of gaze $[F(1,3)=11.86, p=0.041]$. This confirms the observation shown in Fig.3-A/ B that with visible gaze the time of recognition was more variable. The average values of distance, orientation, and time for both humanhuman scenarios at the moment of goal recognition are given in table III. The distance between the approaching person and the test subject at the moment of goal recognition is depicted for both scenarios and each subject in Fig.5. Note the large interindividual differences in goal recognition ranging from almost $5 \mathrm{~m}$ to less than $3 \mathrm{~m}$, which, however, are not influenced by gaze visibility. A repeated measures ANOVA on the distance between approaching agent and subject at the moment of goal recognition showed a significant main effect of subject position $[F(2,3)=13.88, p=0.03]$ and a significant interaction between target and subject position $[F(4,6)=5.67, p=0.03]$. The main effect was due to a larger distance when the subject was standing at position 1 or 2 compared to position 3 (see Fig.2). The orientation of the approaching subject at the moment of goal recognition (see Fig.1) showed a significant main effect of gaze $[F(1,3)=$ $18.58, p=0.022]$ : if the gaze direction of the approaching subject was visible, his orientation was on average turned further away from the subject (table III). Further significant main effects were found for subject position $[F(2,3)=$ $229.88, p<0.001]$ and target position $[F(2,6)=12.5, p=$ 0.007]. In Fig. 4 the orientation angles when approaching the subject and when approaching another target are shown for the gaze (Fig.4-A) and no gaze scenario (Fig.4-B).

\section{B. Human-Human and Human-Robot Experiments}

A representative example of one human-robot experimental session is shown in Fig.2-B. Note that the robot trajectories do not show oscillations, which are typical in humans. 

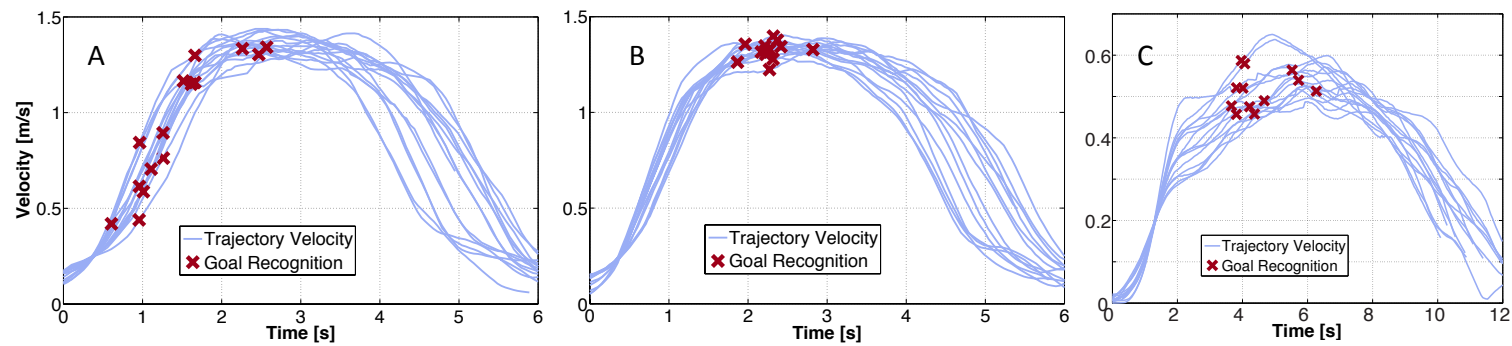

Fig. 3. Velocity profiles with the corresponding goal recognition time stamp (red cross) for a human-human experimental session under normal conditions $\mathbf{A}$ and without visible gaze $\mathbf{B}$. $\mathbf{C}$ represents a human-robot experimental session.

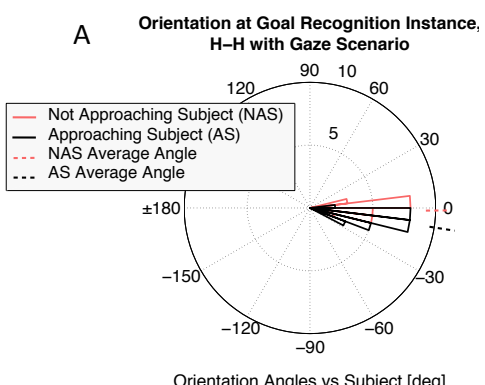

Orientation Angles vs Subject [deg]

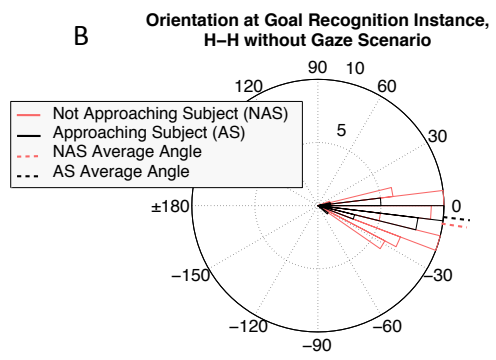

Orientation Angles vs Subject [deg]

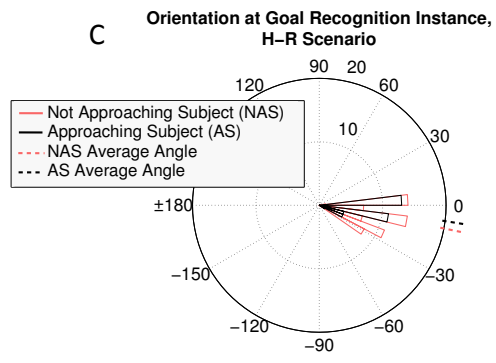

Orientation Angles vs Subject [deg]

Fig. 4. A Orientation angle with respect to the subject at the moment of goal recognition for the human-human scenario with visible gaze. B Orientation angles with respect subject at the moment of goal recognition for the human-human scenario without visible gaze. C Orientation angles vs subject at the moment of goal recognition for the human-robot scenario.
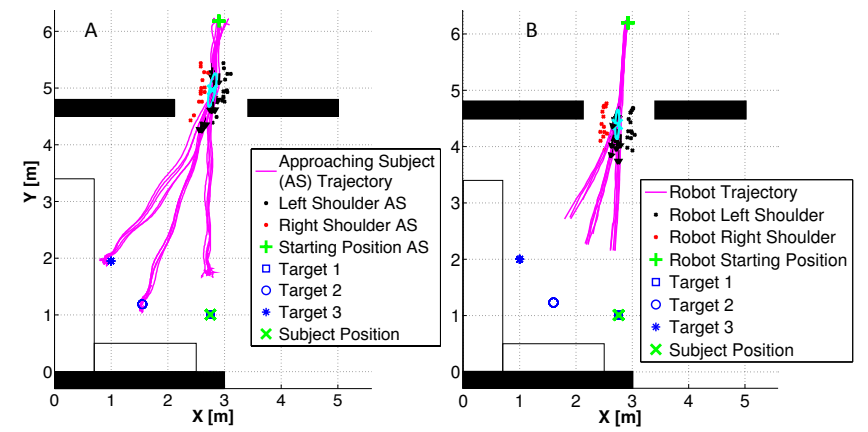

Fig. 2. A Representative human-human experimental session without gaze visibility. The red and black dots represent the right and left shoulder of the approaching person. The midpoint of the shoulders is depicted by a black arrow, which corresponds to the walking direction (orientation). The cyan ellipse is the covariance ellipse of the midpoints scaled to standard deviation. The magenta curves represent the trajectories of the midpoint between the tracked shoulders of the 15 performed approaches. B Representative humanrobot experimental session..

The velocity profiles of one session (15 trajectories) are shown in Fig.3-C. A comparison with the corresponding figure for the human-human scenarios shows that even though the robot moved with a much lower maximum velocity, the moment of goal recognition was at approximately the same relative location in the trajectory. Corresponding to Fig.5 for the human-human experiments, Fig.6 shows the distance between subject and robot for the human-robot scenario at the moment of goal recognition. As for the human-human scenario, the average distance was at about
TABLE III

DISTANCE BETWEEN APPROACHING AGENT AND SUBJECT, ORIENTATION WHEN APPROACHING THE SUBJECT OR ANOTHER TARGET AND TIME LEFT TO REACH THE TARGET AT THE MOMENT OF THE GOAL ATTRIBUTION.

\begin{tabular}{lll}
\hline Parameters & Gaze & No Gaze \\
\hline Distance [m] & $3.9 \pm 0.86$ & $3.8 \pm 0.65$ \\
Orientation [deg] & $-11.4 \pm 13$ & $-8.5 \pm 12.2$ \\
Time to Target [s] & $3.76 \pm 1.44$ & $3.93 \pm 1.22$ \\
\hline
\end{tabular}

$4 \mathrm{~m}$. The orientation angle for the human-robot scenario is shown in 4-C. As in the human-human scenario, the range increased when the robot approached a target instead of the subject (see table IV). Table IV compares average values for agent orientation and subject-agent distance for both the human-human (no-gaze) and human-robot scenario. For the human-robot experiments, the repeated measures ANOVA on the distance between approaching agent and subject at the moment of goal attribution showed a significant main effect of subject position $[F(2,7)=4.94, p=0.045]$ similar to the one observed for human-human experiments. The orientation of the approaching agent at the moment of goal recognition was significantly different depending on whether the agent was a human or the robot $[F(1,7)=16.31, p=0.004]$ with the robot being turned further away from the subject than the human (table IV). Further significant main effects were found for subject position $[F(2,7)=279.2, p<0.0001]$ and for target position $[F(2,7)=7.09, p=0.007]$. 


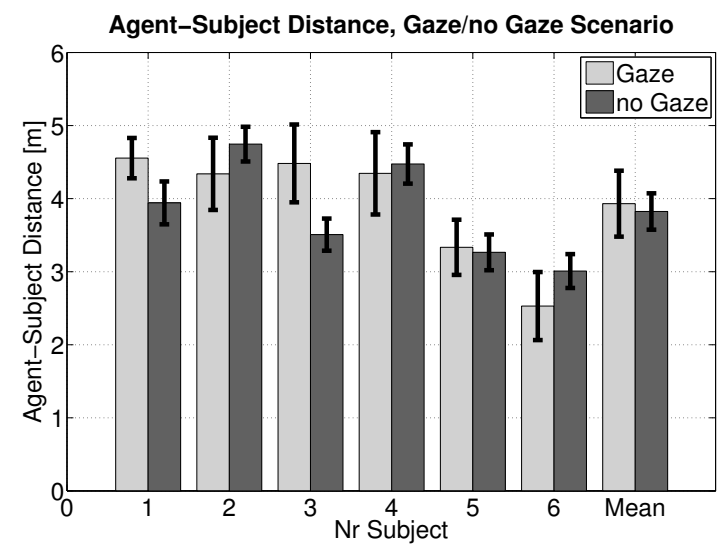

Fig. 5. Mean and standard deviation of the interpersonal distance between the approaching person and the subject at the moment of goal recognition.

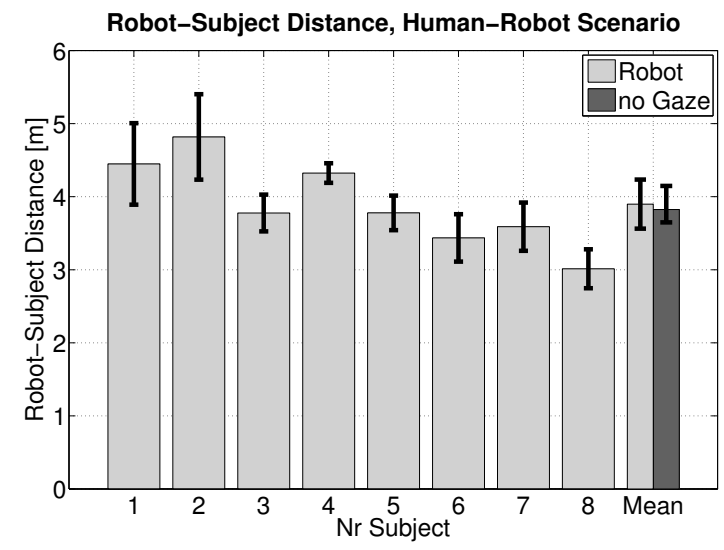

Fig. 6. Mean and standard deviation of the distance between the approaching robot and the subject at the moment of the goal recognition.

\section{DISCUSSION}

Intention recognition in humans constitutes a key factor for a smooth and efficient interaction [17]. While intentions refer to long-term planning, it is often crucial to recognize the short-term goal of an action. In the present study, we could show that inferring the goal of an approaching human can be done with great accuracy and from a relatively large distance, which was about $4 \mathrm{~m}$ in the present study. As expected, this ability deteriorated when the gaze direction of the approaching human was disguised. When the approaching human was replaced by an approaching wheeled robot, the error rate increased to $19 \%$, even though the distance (but

TABLE IV

DISTANCE BETWEEN APPROACHING AGENT AND SUBJECT, ORIENTATION WHEN APPROACHING THE SUBJECT OR ANOTHER TARGET AT THE GOAL RECOGNITION INSTANCE.

\begin{tabular}{lll}
\hline Parameters & No Gaze & Robot \\
\hline Distance [m] & $3.8 \pm 0.65$ & $3.89 \pm 0.6$ \\
Orientation [deg] & $-8.5 \pm 12.2$ & $-10 \pm 11$ \\
\hline
\end{tabular}

not the time) at which subjects attributed the goal remained constant.

\section{A. Human-Human Experiments}

The comparison between human-human experiments with and without visible gaze suggests that subjects utilized gaze direction as an efficient cue when inferring the locomotor goal of a person. This finding was expected, since previous studies had demonstrated that head direction precedes walking direction (e.g. [7]) and that, in an obstacle-free environment, people direct their fixations primarily ahead or at the goal $(75 \%)$ [18]. It is likely that humans uses additionally cues for inferring the locomotion goal of a person, but gaze might be the most important cue because of its predictive behavior [2]. A reason for the predictive nature of gaze direction is its role in supporting the execution of motor actions [19]. Gaze provides the information needed for performing a task and is therefore directed to the relevant objects or goals before the action itself takes place. In a previous investigation on human-human interaction, we could show that an approach followed by a handover is executed in smoothly by blending the two components into each other rather than executing them serially [20]. This efficiency can be attributed to the ability to mutually predict the actions of each other with high accuracy and thus to be able to rely on these predictions. Disguising gaze direction led to significantly more errors in goal recognition, but did not significantly change the location at which the decision was taken. Nonetheless, the variability of the person's location at which the goal was recognized was larger when gaze was visible, because in several trials subjects recognized the goal very early, while that was only rarely the case for the no-gaze condition. Locomotion studies also demonstrated a close relationship between instantaneous velocity and curvature [21], similar to the two-thirds power law underlying handwriting and drawing movements [22]: when curvature of the trajectory is low, the movement is faster. This lawful relation might underlie another finding of our study: most decisions, especially when gaze as reliable cue was absent, were done when the approaching agent had already reached his peak velocity, since high velocity corresponds to a small likelihood of changing direction. The effects of other cues on goal inference, e.g. posture, head movement, arm and leg swing will be investigated in further studies.

\section{B. Human-Robot Experiments}

When the approaching agent was a wheeled robot, which moved with a human-inspired trajectory and velocity profile, we observed a 3-fold increase in error rate compared to the no-gaze human-human scenario. Notably, goal recognition occurred at the same location in space, but did not depend on time to reach the goal, since the robot only could move at about half the speed of the human. While the missing head and eye movements of the robot evidently cannot account for the increase in errors, a multitude of other differences between human and robot ranging from peak velocity to appearance may contribute to the low recognition rate. Due to 
the limitations of the robot system, a direct comparison of the human-human and human-robot experiments is ambiguous. Human-like movements performed by a robotic agent evoke responses similar to those caused by an interacting person. This leads to anthropomorphising [23] and attributing intentions to technical systems [24]. A robotic assistant should therefore behave in a human-like way in order to facilitate the recognition of its actions. As an example, we have previously shown that interaction during a handover was more effective when the robots's movement was performed using a biological minimum-jerk trajectory [25]. The appearance of a robotic agent becomes more relevant only when the robot must execute more complex tasks [26]. In the present case, the decision of our subjects to have recognized the goal of the robot's movement happened at the same location as in our human-human experiments. Thus, the behavior of the robot apparently convinced subjects that the quality of their goal recognition ability was unchanged, even though the sensory cues were not sufficient. In further studies the robot systems will be equipped with an artificial head and eyes to investigate if eye and head cues can reduce the error rate in the goal inference task.

\section{Conclusions}

The present paper investigated the ability to attribute and recognize a goal to an approaching agent. In our scenario, recognition rate was close to $100 \%$ for a human. We also showed that the visibility of the gaze direction of the approaching person plays an important role in goal recognition: when gaze direction was hidden, performance decreased to about $95 \%$. Finally, replacing the human by a wheeled robot moving on a comparable trajectory deteriorated goal recognition to about $80 \%$. To avoid errors in goal recognition, our subjects simply could have waited for a longer time to collect further evidence in favor of a decision from observing the path of the approaching agent. However, apparently our subjects were not aware of their increased error rates but erroneously relied on their ability to correctly recognize the goal of an agent's movement. In a real workplace scenario such a behavior, which is not optimal in the sense of decision theory [27], could lead to inefficient cooperation due to the need for frequent re-planning and even to dangerous situations such as collisions. The experimental results of the present work will build the basis to develop and test goal attributed motion planners.

\section{ACKNOWLEDGMENT}

This work is supported by the DFG cluster of excellence CoTeSys (www.cotesys.org).

\section{REFERENCES}

[1] Rizzolatti G, Craighero L (2004) The mirror-neuron system. Annual Reviews of Neuroscience, 27: 661-670

[2] Flanagan J R, Johansson R S (2003) Action plans used in action observation. Nature, 424: 769-771

[3] Iacoboni M, Molnar-Szakacs I, Gallese V, Buccino G, Mazziotta J C, Rizzolatti G (2005) Grasping the intentions of others with one's own mirror neuron system. Public Library of Science Biology, 3: e79
[4] Calvo-Merino B, Grzes J, Glaser D E, Passingham R E, Haggard P (2006) Seeing or Doing? Influence of Visual and Motor Familiarity in Action Observation. Current Biology, 16: 1905-1910

[5] Goldman A (1992) In defense of the simulation theory. Mind \& Language, 7, 104-119

[6] Frith C, Frith U (2005) Theory of mind. Current Biology, 15(17), R644-R645

[7] Grasso R, Glasauer S, Takei Y, Berthoz A (1996): The predictive brain: anticipatory control of head direction for the steering of locomotion. Neuroreport 7:1170-1174

[8] Demiris Y (2007) Prediction of intent in robotics and multi-agent systems. Cognitive Processing, 8(3), 151-158

[9] Mitsunaga N, Smith C, Kanda T, Ishiguro H, Hagita N (2008) Adapting Robot Behavior for HumanRobot Interaction. IEEE Transactions on Robotics, 24(4), 911-916

[10] Kamewari K, Kato M, Kanda T, Ishiguro H, Hiraki K (2005) Six-anda-half-month-old children positively attribute goals to human action and to humanoid-robot motion. Cognitive Development, 20: 303-320

[11] Arechavaleta G, Laumond J P, Hicheur H, Berthoz A (2008) On the nonholomic nature of human locomotion. Autonomous Robots, 25 , 25-35

[12] Sisbot E A, Marin-Urias L F, Alami R, Simeon T (2007) A Human Aware Mobile Robot Motion Planner. IEEE Transactions on Robotics, 23(5), 874-883

[13] Shi D, Collins Jr E G, Donate A, Liu X, Goldiez B, Dunlap D (2008) Human-aware robot motion planning with velocity constraints. International Symposium on Collaborative Technologies and Systems (CTS): 490-497

[14] Kirsch A, Kruse T, Moesenlechner L (2009) An Integrated Planning and Learning Framework for Human-Robot Interaction. 4th Workshop on Planning and Plan Execution for Real-World Systems (held in conjuction with ICAPS 09)

[15] Althoff D, Kourakos O, Lawitzky M, Mörtl A, Rambow M, Rohrmller F, Brščić D, Wollherr D, Hirche S and Buss M (2009) A flexible architecture for real-time control in multi-robot systems. Proceedings of the Third International Workshop on Human Centered Robotic Systems '09 , Cognitive Systems Monographs, Springer: 43-52

[16] Ulrich R, Mattes S, Miller J (1999) Donders's assumption of pure insertion: an evaluation on the basis of response dynamics. Acta Psychologica, 102(1): 43-75

[17] Sebanz N, Knoblich G (2009) Prediction in Joint Action: What, When, and Where. Topics in Cognitive Science, 1(2): 353-367

[18] Turano K A, Geruschat D R, Baker F H, Stahl J W, Shapiro M D (2001) Direction of gaze while walking a simple route: persons with normal vision and persons with retinitis pigmentosa. Optometry and Vision Science, 78(9), 667-675

[19] Land M F (2006) Eye movements and the control of actions in everyday life. Progress in Retinal and Eye Research 25(3), 296-324

[20] Basili P, Huber M, Brandt T, Hirche S, Glasauer S (2009) Investigating Human-Human Approach and Hand-Over. Proceedings of the Third International Workshop on Human Centered Robotic Systems '09, Cognitive Systems Monographs, Springer, 151-160

[21] Hicheur H, Vieilledent S, Richardson M J E, Flash T,Berthoz A (2005) Velocity and curvature in human locomotion along complex curved paths: a comparison with hand movements. Exp Brain Res, 162(2), 145-154

[22] Viviani P, Terzuolo C (1982) Trajectory determines movement dynamics. Neuroscience, 7, 431-437

[23] Duffy B R (2003) Anthropomorphism and the social robot. Robotics and Autonomous Systems, 42, 177-190

[24] Dennett D C (1987) The Intentional Stance. Cambridge MA. MIT Press

[25] Huber M, Rickert M, Knoll A, Brandt T, Glasauer S. Human-Robot Interaction in Handing-Over Tasks. Proc 17th IEEE Int Symp on Robot and Human Interactive Communication, pp. 107-112, 2008

[26] Walters L M, Syrdal D G, Dautenhahn K, Boekhorst R, Koay K L (2008) Avoiding the uncanny valley: robot appearance, personality and consistency of behavior in an attention-seeking home scenario for a robot companion. Autonomous Robots 24(2), 159-178

[27] Bather J, (2000) Decision theory: an introduction to dynamic programming and sequential decisions. Wiley Chichester 